\title{
SARS-CoV-2 and the safety margins of cell-based biological medicinal products
}

\author{
Jens Modrof ${ }^{1}$, Astrid Kerschbaum ${ }^{2}$, Maria Farcet ${ }^{2}$, Daniela Niemeyer ${ }^{3}$, Victor Corman ${ }^{3}$, \\ and Thomas Kreil ${ }^{4}$ \\ ${ }^{1}$ Takeda Pharmaceutical Co Ltd \\ ${ }^{2}$ Baxter AG Austria \\ ${ }^{3}$ Charité Universitätsmedizin Berlin \\ ${ }^{4}$ Takeda Austria Vienna
}

June 3, 2020

\begin{abstract}
With the pandemic emergence of SARS-CoV-2, the exposure of cell substrates used for manufacturing of medicines has become a possibility. Cell lines used in biomanufacturing were thus evaluated for their SARS-CoV-2 susceptibility, and the detection of SARS-CoV-2 in culture supernatants was tested by routine adventitious virus testing of fermenter harvest.
\end{abstract}

\section{Introduction}

A general concern in cell-based manufacturing of recombinant proteins, including vaccines, is the potential contamination of the cell culture with viruses, which has had severe consequences for patients and manufacturers (Barone et al., 2020; Berting, Farcet, \& Kreil, 2010). With the pandemic emergence of SARS$\mathrm{CoV}-2$, the exposure of biomanufacturing cell lines to a new virus has become a possibility, and to safeguard biomedicines it is important to understand whether the virus is even capable of infecting bioproduction cell lines, and if so, whether the event would be detected by adventitious agent tests (AAT) that are part of biomanufacturing quality control.

This study investigated four widely used biomanufacturing cell lines, i.e. CHO and Vero, as well as the two human cell lines HEK-293 and HT-1080, for their susceptibility to infection with SARS-CoV-2.

In addition, adventitious virus testing (AAT) as routinely performed for in-process control testing per regulatory guidance (US Food and Drug Administration, 1993; ICH, 1997), was evaluated for its ability to detect SARS-CoV-2 infection if it were to occur. As MRC-5 cells are part of the AAT detector cell line panel (Figure 1), these cells were also tested for their susceptibility to SARS-CoV-2.

\section{Materials and Methods}

\subsection{Virus and Cells}

SARS-CoV-2 strain BetaCoV/Germany/BavPat1/2020 (kindly provided by Charité Universitätsmedizin, Institute of Virology, Berlin, Germany; EVAg 026V-03883) was used for all inoculations.

CHO cells (Chinese hamster ovary, ATCC CCL-61), Vero cells (African green monkey kidney epithelial, CCL-81, sourced from the ECACC 84113001), HEK-293 cells (Human embryonic kidney cell line, ATCC CRL-1573), HT-1080 cells (Human fibrosarcoma cell line, ATCC CCL-121), and MRC-5 cells (human lung fibroblast, ATCC CCL-171) were used. 


\subsection{Inoculation of Cells With SARS-CoV-2 and Adventitious Agent Testing}

Cells (CHO, Vero, HEK-293, HT-1080 and MRC-5) in 6-well plates were incubated with SARS-CoV-2 for 14 days. Inoculations with virus were done at multiplicity of infection $(\mathrm{MOI})=0.01$ and $\mathrm{MOI}=1$, that is, approx. $10^{3}$ and $10^{5}$ infectious virus particles per well, respectively, for $1 \mathrm{~h}$. Then the respective medium was added to a total volume of $6 \mathrm{~mL} /$ well (CHO medium: Ham's F12 medium supplemented with 10\% FCS [PAN-Biotech P40-2009], L-glutamine [2mM], nonessential amino acids [1x], sodium pyruvate $[1 \mathrm{mM}]$ and Gentamycin sulfate $[100 \mathrm{mg} / \mathrm{ml}]$ ); Vero medium: TC-Vero medium supplemented with $5 \% \mathrm{FCS}$, L-glutamine $[2 \mathrm{mM}]$, nonessential amino acids $[1 \mathrm{x}]$, sodium pyruvate $[1 \mathrm{mM}]$, Gentamycin sulfate $[100 \mathrm{mg} / \mathrm{ml}]$ and sodium bicarbonate [7.5\%]; HEK 293 medium: EAGLE-MEM (+Earles BSS) supplemented with 5\% FCS and Gentamycin sulfate $[100 \mathrm{mg} / \mathrm{ml}]$; HT-1080 medium: McCoy 5 A supplemented with $2 \%$ FCS , L-glutamine $[2 \mathrm{mM}]$, sodium pyruvate $[1 \mathrm{mM}]$ and Gentamycin sulfate $[100 \mathrm{mg} / \mathrm{ml}]$; MRC- 5 medium: EAGLE-MEM (+Earles BSS) supplemented with $10 \%$ FCS, L-glutamine [2mM], nonessential amino acids [1x], sodium pyruvate $[1 \mathrm{mM}]$, Gentamycin sulfate $[100 \mathrm{mg} / \mathrm{ml}]$ and sodium bicarbonate [7.5\%]). 6-well plates were incubated for 14 days at $36^{\circ} \mathrm{C}$ and $4.5 \% \mathrm{CO}_{2}$. The presence of cytopathological effects (CPE) was assessed on days 2, 3, 6, 7 and 14 post-infection.

The presence of infectious SARS-CoV-2 was tested by titration at various stages throughout the incubation period, on Vero cells using a TCID $_{50}$ assay (see below). Samples for titration were taken from the input inoculum and days 2 (Vero cells MOI=1 only), 3, 6, 7 and 14 post-infection.

For each cell line, inoculations with SARS-CoV-2 at the two MOIs was done in duplicate.

In addition to SARS-CoV-2 readout by CPE, after 14 days of inoculation, hemadsorption (HAD) and hemagglutination (HA) assays were done, as routinely performed during AAT.

For HAD, wells with MRC-5, Vero and CHO cells were covered with erythrocyte suspensions of two different species (human $0.5 \%[\mathrm{v} / \mathrm{v}]$ and guinea pig $1 \%[\mathrm{v} / \mathrm{v}]$ ). Separate plates were incubated at $+2-8^{\circ} \mathrm{C}$ and $36^{\circ} \mathrm{C}$ for $30 \mathrm{~min}$, the cell culture supernatant removed, and the cells washed twice with PBS before microscopic inspection for HAD.

For HA, supernatants of MRC-5, Vero, CHO, HEK-293 and HT-1080 cells were diluted with 0.9\% [w/v] $\mathrm{NaCl}$ solution in twofold steps. Erythrocyte suspensions (human, $0.5 \%[\mathrm{v} / \mathrm{v}]$ and guinea pig $1.0 \%[\mathrm{v} / \mathrm{v}]$ ) were added and separate $\mathrm{V}$-shaped plates incubated for $35 \mathrm{~min}$ at $+2-8^{\circ} \mathrm{C}$ and $36^{\circ} \mathrm{C}$, before hemagglutination was inspected visually. The described AAT is essentially the routine procedure used for the testing of recombinant protein bulk harvests.

\subsection{Infectivity assay $\left(\right.$ TCID $\left._{50}\right)$}

Samples that potentially contained infectious virus were titrated by tissue culture infectious dose $50 \%$ $\left(\mathrm{TCID}_{50}\right)$ assay, that is, eightfold replicates of serial half-log sample dilutions were incubated with cells for 5-7 days and CPE was assessed microscopically. Virus concentrations were calculated according to the Poisson distribution and expressed as viral $\operatorname{load}\left(\log _{10}\left[\mathrm{TCID}_{50}\right]\right)$.

\section{Results and Discussion}

Five cell lines (CHO, Vero, HEK-293, HT-1080 and MRC-5) were inoculated in duplicate with SARS-CoV-2 at two different MOI values of 1 and 0.01, respectively, and incubated for 14 days. During the incubation period the cells were periodically observed for CPE, as an indicator for viral infection in the same way as performed during routine AAT.

No CPE was detected throughout the incubation period for CHO cells, HEK-293 cells, HT-1080 cells and MRC-5 cells. In contrast, Vero cells displayed a CPE already on day $2(\mathrm{MOI}=1)$ or day $3(\mathrm{MOI}=0.01)$ post infection (p.i.). The permissiveness of Vero E6 and CCL81 cells for SARS-CoV-2 infection and production of CPE has already been demonstrated (Harcourt et al., 2020), and thus Vero CCL81 cells were used in this study as positive control, as well as for quantitation of SARS-CoV-2 replication in the other cell lines evaluated: the presence of infectious virus in the cell culture supernatant was determined at regular intervals 
throughout the incubation period by $\mathrm{TCID}_{50}$ assay, starting with the inoculum preparations and up to the last day of incubation (day 14).

For Vero cells inoculated with MOIs of 1 and 0.01, this analysis showed increasing SARS-CoV-2 titers in cell supernatants from day 2 and day 3 p.i., which peaked on days 6 and 7 p.i., at mean titers of 6.1 and $5.7 \log _{10} \mathrm{TCID}_{50} / \mathrm{mL}$, and by 14 days p.i. slightly dropped by on average of 0.7 and $0.8 \log _{10}$, respectively (Table 1).

For the CHO, HT-1080 and MRC-5 cell lines, no SARS-CoV-2 infectivity was detected by titration on Vero cells throughout the entire incubation period, which indicates that SARS-CoV-2 cannot replicate in these cells.

For HEK-293 cells inoculated at MOI=0.01, SARS-CoV-2 infectivity was never detectable in culture supernatants. After inoculation at MOI=1, however, low and constantly declining SARS-CoV-2 infectivity titers were detected until day 7 p.i. (Table 1). Whether these declining levels of virus reflect residual inoculum which may be more effectively bound by the HEK-293 as opposed to the CHO, HT-1080 and MRC-5 cell layers, or even some limited yet abortive replication as observed for other HEK-293-derived cells (Harcourt et al., 2020) remains to be determined.

In addition to the monitoring for the development of any CPE, the cells and supernatants were assayed at the end of the 14 days incubation period in the two standard AAT readouts, i.e., HAD to the cells, and HA activity in the supernatant, using human and guinea pig erythrocytes. This panel of altogether three assay readouts (CPE, HAD, HA) reflects exactly what is routinely performed during quality control testing of biomanufacturing fermenter harvests by using at least three different indicator cell lines (Figure 1). The choice of detection cells lines for AAT includes per FDA guidance (i) the type of cells used for biopharmaceutical production, (ii) a human diploid cell line, e.g. MRC-5 cells, and (iii) a monkey kidney cell line, e.g. Vero cells (US Food and Drug Administration, 1993).

For SARS-CoV-2 inoculated CHO cells, HT-1080 cells, HEK-293 cells and MRC-5 cells, virus was not detectable by any of the three AAT readouts, i.e. CPE as monitored throughout the two weeks of incubation and HAD / HA at the end of the incubation period. For Vero cells though, SARS-CoV-2 replication was, in addition to detection by $\mathrm{CPE}$, detected by HAD with human erythrocytes (MOI=1 only). These findings confirm that AAT would confidently detect a SARS-CoV-2 contamination in a biomanufacturing cell culture tested, so long as Vero cells are included as a detector cell line.

Entry of SARS-CoV-2 into susceptible cells is facilitated by the same receptor as for SARS-CoV, i.e. the Angiotensin-converting enzyme-2 (ACE 2) (Hoffmann et al., 2020; Zhou et al., 2020), which suggests that the spectrum of susceptible cells is similar for SARS-CoV and SARS-CoV-2. High abundance of ACE2 as a cellular surface protein on Vero cells (Kaye, 2006) does correlate well with their susceptibility to infection with SARS-CoV-2. In contrast, the expression of ACE2 at low levels on CHO cells (Warner et al., 2005) might be the reason that SARS-CoV-2 replication in these cells was not observed. HEK-293 cells carry ACE2 at higher levels (Warner et al., 2005), which may facilitate binding and possibly entry of SARS-CoV-2 into HEK-293, followed by minimal and ultimately abortive replication (Table 1), or modest replication in HEK-293T cells (Harcourt et al., 2020).

\section{Conclusions}

Permissiveness for infection with SARS-CoV-2 was confirmed for Vero cells, but was absent for CHO, HT1080 and MRC-5 cells. HEK-293 cells did not show productive amplification of SARS-CoV-2 although some minimal and self-limiting replication may be possible. This study also showed that in CHO cells, HT-1080 cells and MRC-5 cells, no "silent" infection of SARS-CoV-2 did occur, i.e. virus replication but no development of detectable CPE, as a "silent" virus replication would have been detected by the titration of supernatants on Vero cells. In routine AAT assays, SARS-CoV-2 was confidently detected by CPE in Vero cells and HAD with human erythrocytes, i.e. two different readouts that revealed the presence of SARS-CoV-2, which underlines its reliable detection by AAT if it were to occur as a contaminant in a biomanufacturing cell culture process. 
The detection of SARS-CoV-2 in AAT when Vero cells are part of the indicator cell line panel was reassuring, even in the hypothetical case that SARS-CoV-2 would replicate in the production cell line but would not produce a CPE and not be detected by cell parameter screening, for example by measurement of cell viability, oxygen consumption etc.

Furthermore, SARS-CoV-2 would represent a contaminant for which highly effective clearance capacities can be expected from widely used dedicated down-stream virus reduction steps: for example, as a lipid-enveloped virus SARS-CoV-2 would be robustly inactivated by solvent/detergent (S/D) treatment (Dichtelmueller et al., 2009) and due to its large size of about $120 \mathrm{~nm}$ SARS-CoV-2 can be expected to be completely retained by nanofiltration, even when using larger pore sizes such as $35 \mathrm{~nm}$ (Burnouf, Radosevich, Goubran, \& Willkommen, 2005).

\section{Acknowledgments}

The contributions of the Global Pathogen Safety team from Takeda, most notably Petra Gruber, Sabine Lehner, Alexandra Schlapschy-Danzinger, Melanie Graf, Eva Ha, Cornelia Lackner, Simone Knotzer, Veronika Sulzer, and Sabrina Brandtner are gratefully acknowledged.

\section{Conflict of Interests}

Jens Modrof, Maria R. Farcet, and Thomas R. Kreil own Takeda shares.

\section{References}

US Food and Drug Administration. (1993). Points to Consider in the Characterization of Cell Lines used to produce Biologicals.

Barone, P. W., Wiebe, M. E., Leung, J. C., Hussein, I. T. M., Keumurian, F. J., Bouressa, J., . . Springs, S. L. (2020). Viral contamination in biologic manufacture and implications for emerging therapies. Nat Biotechnol. doi:10.1038/s41587-020-0507-2

Berting, A., Farcet, M. R., \& Kreil, T. R. (2010). Virus susceptibility of Chinese hamster ovary (CHO) cells and detection of viral contaminations by adventitious agent testing. Biotechnology and Bioengineering, 106(4), 598-607.

Burnouf, T., Radosevich, M., Goubran, H. A., \& Willkommen, H. (2005). Place of Nanofiltration for Assuring Viral Safety of Biologicals. Curr Nanosci, 1, 189-201.

Dichtelmueller, H., Biesert, L., Fabbrizzi, F., Gajardo, R., Groner, A., von Hoegen, I., . . . Poelsler, G. (2009). Robustness of solvent/detergent treatment of plasma derivatives: a data collection from Plasma Protein Therapeutics Association member companies. Transfusion, 49, 1931-1943.

Harcourt, J., Tamin, A., Lu, X., Kamili, S., Sakthivel, S. K., Murray, J., . . Thornburg, N. J. (2020). Severe Acute Respiratory Syndrome Coronavirus 2 from Patient with 2019 Novel Coronavirus Disease, United States. Emerg Infect Dis, 26(6). doi:10.3201/eid2606.200516

Hoffmann, M., Kleine-Weber, H., Schroeder, S., Kruger, N., Herrler, T., Erichsen, S., . . Pohlmann, S. (2020). SARS-CoV-2 Cell Entry Depends on ACE2 and TMPRSS2 and Is Blocked by a Clinically Proven Protease Inhibitor. Cell. doi:10.1016/j.cell.2020.02.052

ICH Topic Q5A. (1997). Note for Guidance on Quality of Biotechnological Products: Viral Safety Evaluation of Biotechnology Products Derived from Cell Lines of Human or Animal Origin. (ICH/295/95).

Kaye, M. (2006). SARS-associated coronavirus replication in cell lines. Emerg Infect Dis, 12(1), 128-133. doi:10.3201/eid1201.050496

Warner, F. J., Lew, R. A., Smith, A. I., Lambert, D. W., Hooper, N. M., \& Turner, A. J. (2005). Angiotensinconverting enzyme 2 (ACE2), but not ACE, is preferentially localized to the apical surface of polarized kidney cells. J Biol Chem, 280(47), 39353-39362. doi:10.1074/jbc.M508914200 
Zhou, P., Yang, X. L., Wang, X. G., Hu, B., Zhang, L., Zhang, W., . . Shi, Z. L. (2020). A pneumonia outbreak associated with a new coronavirus of probable bat origin. Nature, 579(7798), 270-273. doi:10.1038/s41586020-2012-7

Table 1 SARS-CoV-2 titers $\left(\mathrm{TCID}_{50} / \mathrm{mL}\right)$ in supernatants of Vero and HEK-293 inoculated in duplicate at MOIs of 0.01 and 1 , respectively.

\begin{tabular}{lll}
\hline Cell line (MOI) & Days post infection & Days post infection \\
\hline & $\mathbf{0}$ & $\mathbf{2}$ \\
Vero (0.01) & 2.0 & $\mathrm{NT}$ \\
Vero (1) & 4.1 & $5.1 / 5.2$ \\
HEK-293 (0.01) & 2.4 & $\mathrm{NT}$ \\
HEK-293 (1) & 4.5 & $\mathrm{NT}$ \\
MOI, Multiplicity of infection; NT, not tested. & MOI, Multiplicity of infection; NT, not tested. & MOI, Multiplicity of infe \\
\hline
\end{tabular}

Figure 1 Overview of production cell lines evaluated for SARS-CoV-2 susceptibility evaluations and the corresponding detection cell lines that make up the respective AAT panels. The information in boxes below indicate whether SARS-CoV-2 permissiveness was detected and for Vero cells the successful assay readouts.

\begin{tabular}{|c|c|c|c|c|c|}
\hline Production Cell Lines & & \multicolumn{4}{|c|}{ AAT Detection Cell Lines } \\
\hline $\mathrm{CHO}$ & $\Longrightarrow$ & $\mathrm{CHO}$ & MRC-5 & Vero & \\
\hline HT- 1080 & $\Longrightarrow$ & HT- 1080 & MRC-5 & Vero & $\mathrm{CHO}$ \\
\hline HEK-293 & $\longrightarrow$ & HEK-293 & MRC-5 & Vero & \\
\hline 乃 & & 凸 & Љ & Љ & 凸 \\
\hline Not infected by SARS-CoV-2 & & No & No & CPE \& HAD & No \\
\hline
\end{tabular}

